\title{
Applications of the Variational Quantum Monte Carlo Method to the Two-Electron Atoms
}

\author{
Salah Badawi Doma ${ }^{1}$, Nazih Abdelhamid Elnohy², Mahmoud Ahmed Salem ${ }^{2}$ \\ ${ }^{1}$ Department of Mathematics and Computer Science, Faculty of Science, Alexandria University, Alexandria, Egypt \\ ${ }^{2}$ Department of Physics, Faculty of Science, Alexandria University, Alexandria, Egypt
}

Email address:

sbdoma@alexu.edu.eg (S. B. Doma), nazih9@yahoo.com (N. A. Elnohy), mahmoud_ms2008@yahoo.com (M. A. Salem)

\section{To cite this article:}

Salah Badawi Doma, Nazih Abdelhamid Elnohy, Mahmoud Ahmed Salem. Applications of the Variational Quantum Monte Carlo Method to the Two-Electron Atoms. International Journal of High Energy Physics. Vol. 6, No. 2, 2019, pp. 42-53. doi: 10.11648/j.ijhep.20190602.13

Received: November 7, 2019; Accepted: November 28, 2019; Published: December 24, 2019

\begin{abstract}
The variational quantum Monte Carlo method was applied to investigate the ground states of the helium atom and helium like ions with atomic number from 1 to 10 and the first four excited states of the helium atom. Furthermore, the investigation of the ground state of helium, $\mathrm{Li}^{+}$, and $\mathrm{Be}^{2+}$ in a confined impenetrable spherical box. Moreover, the calculation of the ground state of the helium atom in a strong magnetic field using four simple trial wave functions. The trial wave functions consist of usual orbital hydrogen wave functions multiplied by correlation function. Using four different correlation wave functions, we describe the interaction of the two electrons with each other and having a small number of variational parameters.
\end{abstract}

Keywords: Variational Monte Carlo Method, Correlation Functions, Helium Like Ions, Helium Excited States, Confined Helium, Strong Magnetic Field

\section{Introduction}

The most atomic theories arise from the independent electron shell model which assumes that every electron is moving in a field combined of the nucleus and the mean distribution of the other electrons. In this model, the effect of two-electron repulsion neglected. This is leading to inaccurate results for the calculated energies of the helium atom and its ions. The study of the effects of the two-electron correlation has been a subject of interest in atomic physics. The ground state of helium and its ions calculated by using wave functions from the orbital product times correlated function depending on the distance between the two electrons. These wave functions depend on several variable parameters, which should satisfy the variational principle to give improved values for energies. The integration of the functions of inter-electronic distance is difficult so that the topic of electron correlation studied by using numerical methods. One of the most important numerical methods is the variational Monte Carlo (VMC) method [1]. It is based on a combination of two ideas, namely, the variational principle and the Monte Carlo evaluation of integrals using importance sampling based on the Metropolis algorithm [2].

A series of papers have been presented to study the correlated wave functions and their effect on the calculated energies. U. Kleinekathöfer et.al [3] proposed a simple nonvariational wave function of the two-electron atoms. The electron-electron correlation described by an ansatz that has the correct behavior for $r_{12}$ and $r_{12}=\infty$. An additional parameter calculated from a perturbation calculation to the ground-state energies for the helium like atoms with $(Z=$ $1-10$ ) and illustrates the importance of asymptotic behavior and the correlation factor to the values of calculated energies. It is the first successful derivation of a nonvariational wave function for simple two-electron atoms and ions. Because of its simplicity and accuracy, this wave function should prove very useful for calculating the effect of the collective properties of two-electron systems. It should also be possible to extend this treatment to excited states, diatomic molecules and atom surface interactions.

Hiroyuki Nakashima and Hiroshi Nakatsuji [4] solved the Schrödinger equation very accurately for the helium atom and its isoelectronic ions $(Z=1-10)$ with the free iterative complement interaction method followed by the variational principle. They obtained highly accurate wave 
functions and energies of the helium atom and its isoelectronic ions. The calculated energy was correct over 40-digit accuracy.

C. Le Sech [5] developed simple analytic two variational parameters wave functions for the ground state of $\mathrm{H}^{-}$, He, $\mathrm{Li}^{+}, \mathrm{Be}^{2+}$ and $\mathrm{B}^{3+}$ atoms (ions). These functions fulfill the cusp conditions at the singularities of the Coulombic field and include the correct behavior for large inter-particle separations. These functions should be useful for the computation of the processes involving two-electron systems. A further improvement obtained by a term with a third parameter is added to the function, so that more accurate values for the obtained energies.

D. Bressanini and G. Morosi [6] improved highly compact wave functions with a clear physical meaning for the $\mathrm{He}$ atom and He-like isoelectronic ion. The electron-nucleus and electron-electron (Jastrow factor) terms used to satisfy the correct asymptotic behavior for both short and long interparticle distances. Some parameters were chosen to satisfy exactly the cusp conditions. While the others optimized by VMC calculations. They tested the validity of the coalescence wave function approximation. The $Z$ dependence of the optimized parameters makes it possible to write a general form of the wave function, and by using $Z$ as an explicit parameter and four parameters independent of $Z$. They checked the validity of this wave function on the case $Z=30$.

Rabeet Singh Chauhan and Manoj K. Harbola [7] modified Le Sech wave function, which gives very accurate total energies for He-like systems so that both the energies and the densities obtained from the modified wave function are relatively more accurate. An advantage of the method used in this work is its relative computational ease in comparison to the conventional variational method of Hylleraas using many parameters. The accuracy of the resulting densities implies that these can be used with confidence to perform fundamental density functional theory investigations.

Ajit J. Thakkar and Toshikatsu Koga [8] reported variational calculations for the ground states of the helium atom and its isoelectronic ions $\mathrm{H}^{-}, \mathrm{Li}^{+} \ldots \mathrm{Ne}^{8+}$. The calculations used generalized Kinoshita expansions with freely optimized, non-integer powers of the Hylleraas coordinates. One hundred-term expansion of this type leads to better energies than any other expansions in the literature with comparable numbers of terms.

New functional kind has been proposed by Richard Habrovský [9], for helium-like atoms. These functions depend explicitly on interelectronic and hyperspherical coordinates. The ground state energy of the helium atom has been calculated using the variational method with a basis, including a single exponential parameter using hyperspherical coordinates. For the helium atom, the wave functions matched the Kato cusp conditions. The important feature of proposed wave functions is the inclusion of negative powers of $R=\sqrt{r_{1}^{2}+r_{2}^{2}}$ in combination with positive powers of $r_{12}$ into the wave function. He showed that this is a necessary condition for the proposed wave function to be a formal solution of the Schrödinger equation.
In his work, he proposed a new ansatz wave function for the helium-like ion. The energy of the helium atom obtained with the use of hyper-radial coordinates showed that the wave functions are close to the exact solution of the Schrödinger equation. The remarkable property of these functions is the chosen expansion coefficient for the functions of the same type. It was shown in [10] a simple exponential of $R$ describes the behavior of the wave functions fairly well in limit cases $r_{1}=0, r_{2}=0$ or $r_{12}=0$.

An implementation of the Hartree-Fock (HF) method using a Laguerre-based wave function described by A. W. King, A. L. Baskerville, and H. Cox [11] to study the ground state of two-electron atoms in the fixed nucleus approximation, and by comparison with fully correlated energies, used to determine accurate electron correlation energies. A variational parameter included in the wave function rapidly increased the convergence of the energy. The one-electron integrals solved by a series solution and an analytical form found in the two-electron integrals. This method is used to produce accurate wave functions, energies and expectation values for the helium isoelectronic sequence and calculating the critical nuclear charge for binding two electrons within the HF approach. The wave functions are shown to provide accurate nucleus electron cusps and expectation values of the inter-particle distances for all systems considered. These HF energies calculated using a wave function that depends explicitly on the inter-electron distance, were used to calculate accurate correlation energies.

Recently, asymptotic behavior of two-electron expectation values in two-electron excited states studied by Montgomery et.al [12]. Also, Jing Li et.al [13] compared many-body approaches against the helium atom exact solution. Moreover, Mohammad Mostafanejad [14] reviewed the fundamental ideas of the free complement method through its application on both ground and first excited states of the helium atom.

For many-electron atoms, many researchers studied the effect of confinement by impenetrable as well as nonimpenetrable spherical boxes. Most of the studies have considered the case of the helium atom as the simplest fewbody system to study electron correlation effects as a function of the cavity dimension into which they embedded. The effect of confinement on the electron correlation arises due to the Coulomb interaction between the two electrons. Methods such as the variational method, self-consistent, configuration interaction (CI) and quantum Monte Carlo (QMC) methods have also been used to study the properties of the helium atom and its ions confined in an impenetrable spherical box.

An important study has been presented to calculate the compression effects in helium-like atoms $(Z=1-5)$ constrained by hard spherical walls [15]. X. Wen-Fang [16] presented a description of the helium atom under spherical parabolic confinement potential using the adiabatic hyperspherical approach method. The obtained results proved that the energies of a spherical parabolic well are in good agreement with those of an impenetrable spherical box for the larger confined potential radius. 
C. Laughlin and S. I. Chu [17] used Hylleraas-type basis functions, which include the interelectronic distance $r_{12}$, to perform accurate calculations for the ground-state energies of a helium atom confined in the center of a spherical cavity in both cases penetrable and impenetrable cavities. In the case of strong confinement of helium atom embedded in a spherical box with impenetrable walls [18], the timeindependent Schrödinger equation was solved using firstorder Rayleigh-Schrödinger perturbation theory and then the calculations were extended using fifth-order variational perturbation theory. The results provided good alternative approaches for the calculation of the ground state energy for a strongly confined helium atom.

Hartree-Fock method and Gaussian basis sets were used to calculate numerous calculations for atoms in penetrable and impenetrable walls. Mariano Rodriguez-Bautista et.al [19, 20] used Roothaan's approach to solve the Hartree-Fock equations for atoms confined by soft walls using the basis set with correct asymptotic behavior. Also, Toby D. Young et.al [21] used two different basis set methods to calculate atomic energy within the Hartree-Fock theory of the confined helium atom.

Recent progress was reviewed by E. Ley-Koo [22] in the field of confined atoms and molecules. Jacob Katriel et.al [23] revised singly-excited and singlet-triplet pairs of states of two-electron spherically symmetric systems that are degenerate in the absence of inter-electronic repulsion. Also, they studied the two-electron quantum dot confined by either a harmonic potential or by an infinite spherical well. Furthermore, Álvaro Luzón et.al [24] studied the first excited states of the helium atom confined under impenetrable spherical walls. Also, Michael-Adán et.al [25] studied spatial confinements that induce localization or delocalization on the electron density in atoms and molecules. They examined the Shannon entropy in configuration space for the hydrogen atom submitted to different potentials: infinite potential, Coulomb potential, harmonic oscillator, constant potential and dielectric continuum.

Moreover, many research groups have added to the understanding of atomic and molecular systems in strong magnetic fields by applying different HF methods, density functional theory, and CI calculations. X. Wang et.al [26] applied approximate expansions of Gaussian-type functions of $r_{12}$ to replace the integer and half-integer powers of $r_{12}$ in Hylleraas-Gaussian basis and used a full (CI) method to calculate the energies of a helium atom in magnetic fields between 0 and 100 a.u. Most of their results were improved at $10^{-4}$ order in precision compared to the results of the full CI method with a Gaussian basis or with incomplete Hylleraas-Gaussian basis, when the electrons restricted to a small spatial extent in a strong magnetic field.

S. Boblest et.al [27] used the combination of a twodimensional HF method and a diffusion QMC method for a thorough investigation of the ground state configurations of all atoms and ions with $Z=2-10$ except for hydrogen-like systems in strong magnetic fields. They obtained the most comprehensive data set of ground-state configurations as a function of the magnetic field strength to analyze and compare the properties of systems with different core charges.

Accurate theoretical and experimental methods are used to investigate the behavior of atoms in a strong magnetic field. Wuming Zhu and S. B. Trickey [28] used anisotropic Gaussian type orbital basis functions to calculate $\mathrm{H}$ through $\mathrm{C}(1 \leq \mathrm{Z} \leq 6)$ and ions $\mathrm{Li}^{+}, \mathrm{Be}^{+}$and $\mathrm{B}^{+}$in a wide of magnetic field (B) range $(0 \leq \mathrm{B} \leq 2000$ a.u. $)$ which showed an accuracy better than single-electron basis sets. Abdullah Zafar et.al [29] used active laser spectroscopy to make highresolution magnetic field measurements in hydrogen and helium plasmas.

S. B. Doma et.al [30] used the VMC method to calculate the ground state of the helium atom as well as the ground state of hydrogen negative ion in the presence of a magnetic field regime between 0 a.u. and 10 a.u. They used two types of compact and accurate trial wave functions. The results are in good agreement with the exact values. Also, Doma and ElGammal [31] presented a study for helium, $\mathrm{Li}^{+}$and $\mathrm{Be}^{2+}$ ions under the compression effect of a spherical box. They used optimized wave function with five variational parameters. Furthermore, they investigated the total energies of the excited states of the helium atom in a strong magnetic field, taking into account the point of transition from the ground state to the excited states [32]. Moreover, S. B. Doma et.al [33] used the VMC technique to study the lithium atom and its like ions up to $Z=10$ in the presence of a magnetic field. The calculations for the ground and some excited states performed for magnetic field strengths ranging from zero up to 100 a.u. Most of their work depended on the Jastrow correlation wave function to consider the electron-electron interaction.

However, it is always required to find a way to simplify the calculations. Therefore, the purpose of this paper is to solve the Schrödinger equation for two-electron atoms by using different correlation functions to investigate the ground state energy of helium and helium-like ions and the low-lying excited states of the helium atom. Furthermore, we study the confinement effect of the helium atom by impenetrable as well as non-impenetrable spherical boxes. Moreover, we investigate the helium atom in a strong magnetic field. The method and the technique of the variational Monte Carlo were described well in [34].

\section{Hamiltonian}

The Hamiltonian operator for helium like atoms in atomic units, when the nucleus is assumed to be with infinite mass, is given by

$$
H=\frac{-1}{2} \sum_{i=1}^{2} \nabla_{i}^{2}-\sum_{i=1}^{2} \frac{Z}{r_{i}}+\frac{1}{r_{12}}
$$

where $Z$ is the atomic number, $r_{i}=\left|\boldsymbol{r}_{i}\right|$ is the distance between the $i^{\text {th }}$ electron and the nucleus, $r_{12}$ is the distance between the two electrons and $\nabla_{i}^{2}$ is the Laplacian operator of the $i^{\text {th }}$ electron. In Hylleraas coordinates, (1) can be written as follows 


$$
\begin{gathered}
H=-\frac{1}{2} \sum_{i=1}^{2} \frac{\partial^{2}}{\partial r_{i}^{2}}-\sum_{i=1}^{2} \frac{1}{r_{i}} \frac{\partial}{\partial r_{i}}-\sum_{i=1}^{2} \frac{Z}{r_{i}}+\frac{1}{r_{12}}-\frac{\partial^{2}}{\partial r_{12}^{2}}-\frac{2}{r_{12}} \frac{\partial}{\partial r_{12}} \\
-\frac{1}{2} \sum_{i \neq j}^{2} \frac{r_{i}^{2}+r_{i j}^{2}-r_{j}^{2}}{r_{i} r_{i j}} \frac{\partial^{2}}{\partial r_{i} \partial r_{i j}}-\frac{1}{2} \sum_{i=1}^{2} \frac{1}{r_{i}^{2}} \frac{\partial^{2}}{\partial \theta_{i}^{2}}-\frac{1}{2} \sum_{i=1}^{2} \frac{1}{r_{i}^{2} \sin ^{2} \theta_{i}} \frac{\partial^{2}}{\partial \varphi_{i}^{2}}-\frac{1}{2} \sum_{i=1}^{2} \frac{\cot \theta_{i}}{r_{i}^{2}} \frac{\partial}{\partial \theta_{i}} \\
-\left(\frac{r_{2}}{r_{1} r_{12}} \frac{\cos \theta_{2}}{\cos \theta_{1}}+\frac{1}{2} \cot \theta_{1} \frac{r_{12}^{2}-r_{1}^{2}-r_{1}^{2}}{r_{1}^{2} r_{12}}\right) \frac{\partial^{2}}{\partial \theta_{1} \partial r_{12}}-\frac{r_{2}}{r_{1} r_{12}} \frac{\sin \theta_{2}}{\sin \theta_{1}} \sin \left(\varphi_{1}-\varphi_{2}\right) \frac{\partial^{2}}{\partial \varphi_{1} \partial \varphi_{12}}
\end{gathered}
$$

\section{The Trial Wave Functions for the Ground State of Helium and Helium-Like Ions}

Our calculations for the ground-state of helium and its isoelectronic ions are based on using total trial wave function consists of correlation function multiplied by spin function and orbital function, taken from Slater determinant for the ground state of the two-electron atom. Since the Hamiltonian operator (2) does not depend on the spin of the two electrons we are interested only on the orbital part of the trial wave function, which is given by

$$
\Psi_{T}=\psi\left(r_{1}, r_{2}\right) f\left(r_{12}\right)
$$

where

$$
\psi\left(r_{1}, r_{2}\right)=\left(\frac{z^{3}}{\pi}\right) e^{-z\left(r_{1}+r_{2}\right)}
$$

The atomic number $Z$ is replaced in (4) by $z$ which is used here as a variational parameter and is called the effective nuclear charge. For function $f\left(r_{12}\right)$, we present different types of correlation functions. The first of which is given simply by

$$
f_{1}\left(r_{12}\right)=e^{\gamma r_{12}}
$$

The second is the Jastrow correlation function [35] given by

$$
\Psi_{2^{3} S}\left(r_{1}, r_{2}\right)=N\left[\psi_{1 s}\left(r_{1}\right) \psi_{2 s}\left(r_{2}\right)-\psi_{1 s}\left(r_{2}\right) \psi_{2 s}\left(r_{1}\right)\right] f\left(r_{12}\right)
$$

b. The state $2^{1} \mathrm{~S}$ is a para (space-symmetric) state corresponding to the configuration $1 s 2 s$ and its trial wave function is, then, of the form

$$
\Psi_{2^{1} S}\left(r_{1}, r_{2}\right)=N\left[\psi_{1 s}\left(r_{1}\right) \psi_{2 s}\left(r_{2}\right)+\psi_{1 s}\left(r_{2}\right) \psi_{2 s}\left(r_{1}\right)\right] f\left(r_{12}\right)
$$

c. For the $2^{1} \mathrm{P}$ state, which is the lowest para-state corresponding to the configuration $1 s 2 p$, we consider the trial wave function

$$
\Psi_{2^{1} \mathrm{P}}\left(r_{1}, r_{2}\right)=N\left[\psi_{1 s}\left(r_{1}\right) \psi_{2 p m}\left(r_{2}\right)+\psi_{1 s}\left(r_{2}\right) \psi_{2 p m}\left(r_{1}\right)\right] f\left(r_{12}\right)
$$

d. For the $2^{3} \mathrm{P}$ state, which is the lowest ortho state corresponding to the configuration $1 s 2 p$, the trial wave function takes the form

$$
\Psi_{2^{3} \mathrm{P}}\left(r_{1}, r_{2}\right)=N\left[\psi_{1 s}\left(r_{1}\right) \psi_{2 p m}\left(r_{2}\right)-\psi_{1 s}\left(r_{2}\right) \psi_{2 p m}\left(r_{1}\right)\right] f\left(r_{12}\right)
$$

where

$$
\psi_{1 s}(r)=\exp \left(-z_{0} r\right)
$$




$$
\begin{gathered}
\psi_{2 s}(r)=\left(1-z_{i} r / 2\right) \exp \left(-z_{i} r / 2\right) \\
\psi_{2 p m}(r)=r \exp \left(-z_{i} r / 2\right) Y_{1, m}(\theta, \phi), m=0, \pm 1
\end{gathered}
$$

In the above equations $z_{0}$ and $z_{i}$ are variational parameters and $N$ is the normalization constant. $f\left(r_{12}\right)$ is the correlation wave function as described in equations from (5) to (8), with the same variational parameters.

\section{The Hamiltonian and Trial Wave Function for Confined Helium Atom and Ions}

In this section, we study the helium atom under compression effects using the VMC method by using an impenetrable spherical box. Also, we will extend our calculations to include some helium like ions, namely: $\mathrm{Li}^{+}$ and $\mathrm{Be}^{2+}$. The non-relativistic Schrödinger equation for confined two-electron helium-like systems with nuclear charge $Z$ can be written as (in atomic units) [37]

$$
H_{C}=-\frac{1}{2} \sum_{l=1}^{2} \nabla_{i}^{2}-\frac{Z}{r_{1}}-\frac{Z}{r_{2}}+\frac{1}{r_{12}}+V_{\text {conf }}\left(r_{1}, r_{2}\right)
$$

where the confining potential $V_{\text {conf }}\left(r_{1}, r_{2}\right)$ is due to an impenetrable spherical box of radius $r_{c}$ and is given by

$$
V_{\text {conf }}\left(r_{1}, r_{2}\right)=\left\{\begin{array}{c}
0, r_{1}, r_{2}<r_{c} \\
\infty, r_{1}, r_{2} \geq r_{c}
\end{array}\right.
$$

Our goal now is to solve the eigenvalue problem

$$
H_{C} \Psi_{C}=E_{\text {conf }} \Psi_{C}
$$

for the lowest eigenvalue, $E_{\text {conf }}$, by using the following trial wave function

$$
\Psi_{C}=\left(\frac{z^{3}}{\pi}\right) e^{-z\left(r_{1}+r_{2}\right)} f\left(r_{12}\right)\left(1-\frac{r_{1}^{2}}{r_{c}^{2}}\right)\left(1-\frac{r_{2}^{2}}{r_{c}^{2}}\right)
$$

where $f\left(r_{12}\right)$ is the Jastrow correlation wave function and the presence of the cutoff factor

$$
\left(1-\frac{r_{1}^{2}}{r_{c}^{2}}\right)\left(1-\frac{r_{2}^{2}}{r_{c}^{2}}\right)
$$

is to guarantee that the boundary conditions $\Psi_{C}=0$ at $r_{1}=r_{c}$ or $r_{2}=r_{c}$ are satisfied [31].

\section{The Hamiltonian and Trial Wave Function for the Helium Atom in Strong Magnetic Field}

In this section, we assume that the nuclear mass is infinite, and the magnetic field is oriented along the $z$-axis. Hence, the non-relativistic Hamiltonian $H_{M a g}$ for the helium tom in a homogeneous magnetic field can be written as [30]:

$$
H_{M a g}=-\frac{1}{2} \sum_{l=1}^{2} \nabla_{i}^{2}-\frac{Z}{r_{1}}-\frac{Z}{r_{2}}+\frac{1}{r_{12}}+\frac{1}{8} B^{2} \rho^{2}+\frac{B\left(L_{Z}+2 S_{Z}\right)}{2} .
$$

where $B$ is the strength of the magnetic field in atomic units, $\rho^{2}=\left(x_{1}^{2}+y_{1}^{2}\right)+\left(x_{2}^{2}+y_{2}^{2}\right), S_{z}$ is the $z$-component of the total spin, $L_{z}$ is the $z$-component of the total angular momentum, $\frac{B^{2} \rho^{2}}{8}$ is the diamagnetic term, $\frac{B}{2} L_{z}$ is the Zeeman term and $B S_{z}$ represents the spin Zeeman term.

For the ground state of helium $L_{z}=0$ and $S_{z}=0$, and the proposed trial wave function is presented as follows

$$
\Psi_{M a g}=\left(\frac{z^{3}}{\pi}\right) e^{-z\left(r_{1}+r_{2}\right)} e^{-\xi \frac{B}{4}\left(\rho_{1}^{2}+\rho_{2}^{2}\right)} f\left(r_{12}\right)
$$

where $e^{-\xi \frac{B}{4}\left(\rho_{1}^{2}+\rho_{2}^{2}\right)}$ is the lowest Landau orbital wave function with $\xi$ as a variational parameter.

\section{Results}

\subsection{The Ground State of Helium}

The results of calculating the ground state energies of the helium and helium like ions by using the first trial wave function showed that the best value of the energy obtained for $(z=Z-0.14)$ and $(\gamma=0.26)$.

The variation of the ground state energy of helium with respect to the two variational parameters $z$ and $\gamma$ of the first trial wave function is shown in Figure 1.

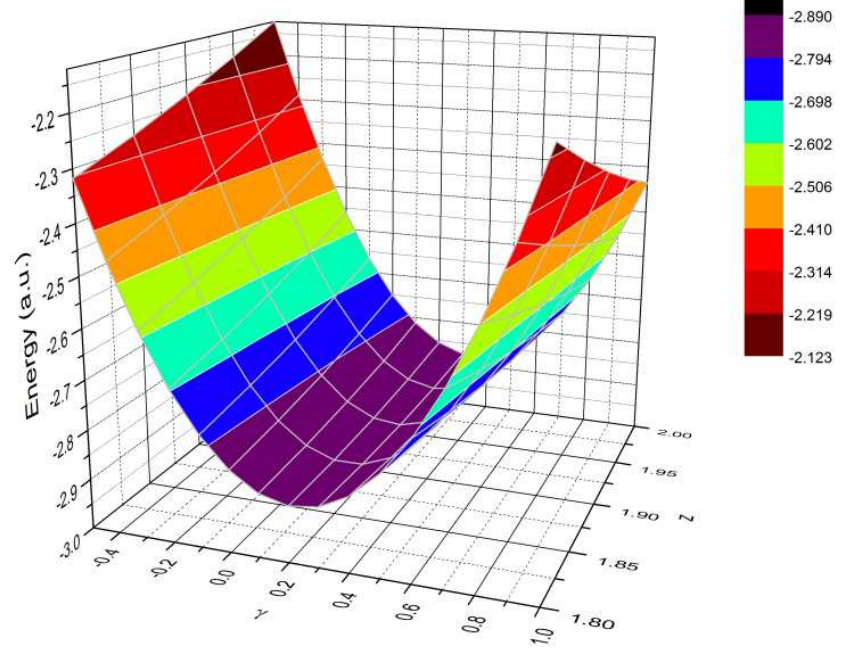

Figure 1. Variation of the ground state energy of helium with respect to the variational parameters by using the first trial wave function. Energy on $Z$ axis, $\gamma$ on $X$-axis and the parameter $Z$ on the $Y$-axis.

In Table 1 we present the values of the parameters of the $2^{\text {nd }}, 3^{\text {rd }}$ and $4^{\text {th }}$ trial wave functions for which the best values of the ground state energy of helium and helium-like ions obtained. $(z=Z-0.1564)$.

Table 1. The best values of the parameters for helium and helium-like ions using 2 nd, 3rd and 4th trial wave functions.

\begin{tabular}{lllll}
\hline $\mathbf{Z}$ & $\mathbf{Z}$ & $\boldsymbol{\beta}$ & $\boldsymbol{\mu}$ & $\boldsymbol{\lambda}$ \\
\hline 1 & 0.8436 & 0.17 & -0.03 & -0.05 \\
2 & 1.8436 & 0.35 & 0.11 & 0.22 \\
3 & 2.8436 & 0.52 & 0.23 & 0.50 \\
4 & 3.8436 & 0.62 & 0.35 & 0.60 \\
5 & 4.8436 & 0.75 & 0.50 & 0.90 \\
\hline
\end{tabular}




\begin{tabular}{lllll}
\hline $\mathbf{Z}$ & $\mathbf{Z}$ & $\boldsymbol{\beta}$ & $\boldsymbol{\mu}$ & $\boldsymbol{\lambda}$ \\
\hline 6 & 5.8436 & 0.97 & 0.75 & 1.70 \\
7 & 6.8436 & 1.50 & 0.91 & 1.86 \\
8 & 7.8436 & 1.90 & 1.10 & 1.88 \\
9 & 8.8436 & 2.21 & 1.18 & 1.90 \\
10 & 9.8436 & 2.42 & 1.28 & 1.92 \\
\hline
\end{tabular}

In Table 2 we present the calculated energies $(E)$, in atomic units, and their associated standard deviation $(\sigma)$ for helium and helium-like ions using the four different trial wave functions. The corresponding exact energies are shown in this table.

Table 2. Calculated energies $(E)$, and standard deviation $(\sigma)$, compared with exact energies for helium and helium-like ions using the different correlation wave functions.

\begin{tabular}{|c|c|c|c|c|c|}
\hline$Z$ & $E^{1}($ a.u. $) \sigma$ & $E^{2}($ a.u. $) \sigma$ & $E^{3}($ a.u. $) \sigma$ & $E^{4}($ a.u. $) \sigma$ & $E^{5}($ a.u. $)$ \\
\hline \multirow{2}{*}{1} & -0.506800 & -0.527689 & -0.508988 & -0.508972 & \multirow{2}{*}{-0.527751} \\
\hline & $1.60 \times 10^{-5}$ & $1.60 \times 10^{-5}$ & $1.50 \times 10^{-5}$ & $1.50 \times 10^{-5}$ & \\
\hline \multirow{2}{*}{2} & -2.889717 & -2.903591 & -2.890321 & -2.890372 & \multirow{2}{*}{-2.903724} \\
\hline & $4.10 \times 10^{-5}$ & $3.70 \times 10^{-5}$ & $3.70 \times 10^{-5}$ & $3.70 \times 10^{-5}$ & \\
\hline \multirow{2}{*}{3} & -7.266911 & -7.279808 & -7.267239 & -7.267313 & \multirow{2}{*}{-7.279913} \\
\hline & $6.60 \times 10^{-5}$ & $5.80 \times 10^{-5}$ & $5.90 \times 10^{-5}$ & $5.90 \times 10^{-5}$ & \\
\hline \multirow{2}{*}{4} & -13.642842 & -13.655489 & -13.642899 & -13.642705 & \multirow{2}{*}{-13.655566} \\
\hline & $9.10 \times 10^{-5}$ & $8.20 \times 10^{-5}$ & $8.10 \times 10^{-5}$ & $8.40 \times 10^{-5}$ & \\
\hline \multirow{2}{*}{5} & -22.018364 & -22.030883 & -22.018444 & -22.018612 & \multirow{2}{*}{-22.030972} \\
\hline & $1.16 \times 10^{-4}$ & $1.04 \times 10^{-4}$ & $1.03 \times 10^{-4}$ & $1.05 \times 10^{-4}$ & \\
\hline \multirow[b]{2}{*}{6} & -32.394005 & -32.406193 & -32.393139 & -32.393543 & \multirow{2}{*}{-32.406247} \\
\hline & $1.41 \times 10^{-4}$ & $1.25 \times 10^{-4}$ & $1.22 \times 10^{-4}$ & $1.20 \times 10^{-4}$ & \\
\hline \multirow{2}{*}{7} & -44.768887 & -44.780988 & -44.768593 & -44.769543 & \multirow{2}{*}{-44.781445} \\
\hline & $1.65 \times 10^{-4}$ & $1.41 \times 10^{-4}$ & $1.43 \times 10^{-4}$ & $1.45 \times 10^{-4}$ & \\
\hline \multirow{2}{*}{8} & -59.144550 & -59.155899 & -59.143051 & -59.144581 & \multirow{2}{*}{-59.156595} \\
\hline & $1.89 \times 10^{-4}$ & $1.58 \times 10^{-4}$ & $1.64 \times 10^{-4}$ & $1.69 \times 10^{-4}$ & \\
\hline \multirow{2}{*}{9} & -75.519073 & -75.531621 & -75.519440 & -75.520035 & \multirow{2}{*}{-75.531712} \\
\hline & $2.17 \times 10^{-4}$ & $1.79 \times 10^{-4}$ & $1.87 \times 10^{-4}$ & $1.95 \times 10^{-4}$ & \\
\hline \multirow{2}{*}{10} & -93.894539 & -93.906744 & -93.894531 & -93.895332 & \multirow{2}{*}{-93.906807} \\
\hline & $2.38 \times 10^{-4}$ & $1.98 \times 10^{-4}$ & $2.10 \times 10^{-4}$ & $2.23 \times 10^{-4}$ & \\
\hline
\end{tabular}

1. Using $1^{\text {st }}$ correlation. 2. Using $2^{\text {nd }}$ correlation. 3. Using $3^{\text {rd }}$ correlation. 4. Using $4^{\text {th }}$ correlation. 5. Exact values [8].

In Figure 2 we present the variation of the ground state energy of helium with respect to the two variational parameters $z$ and $\beta$, by using the second trial wave function.

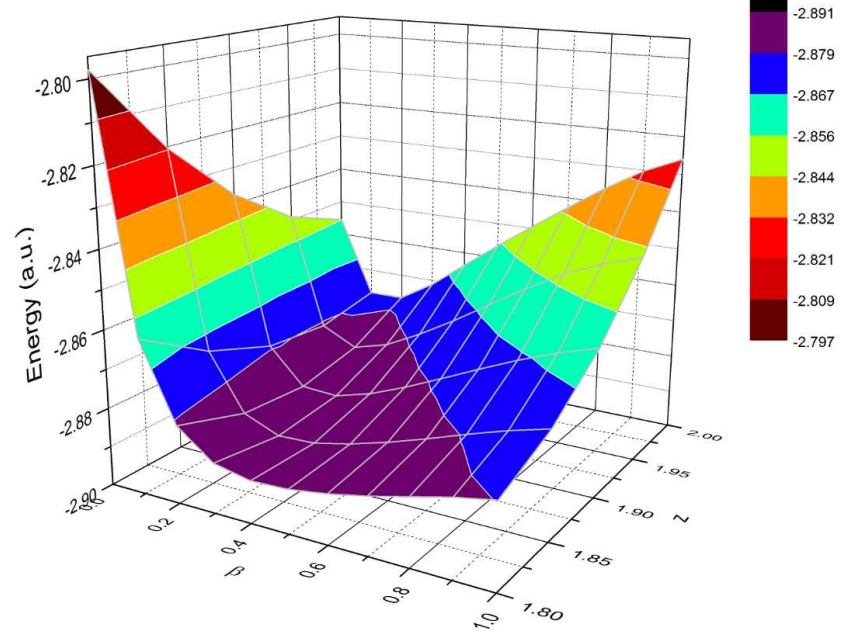

Figure 2. Variation of the ground state energy of helium with respect to the variational parameters, using the second trial wave function. Energy on $Z$ axis, $\beta$ on $X$-axis and the parameter $Z$ - on $Y$-axis.

In Figure 3 we present the variation of the ground state energy of helium with respect to the parameters $z$ and $\mu$, by using the third trial wave function.

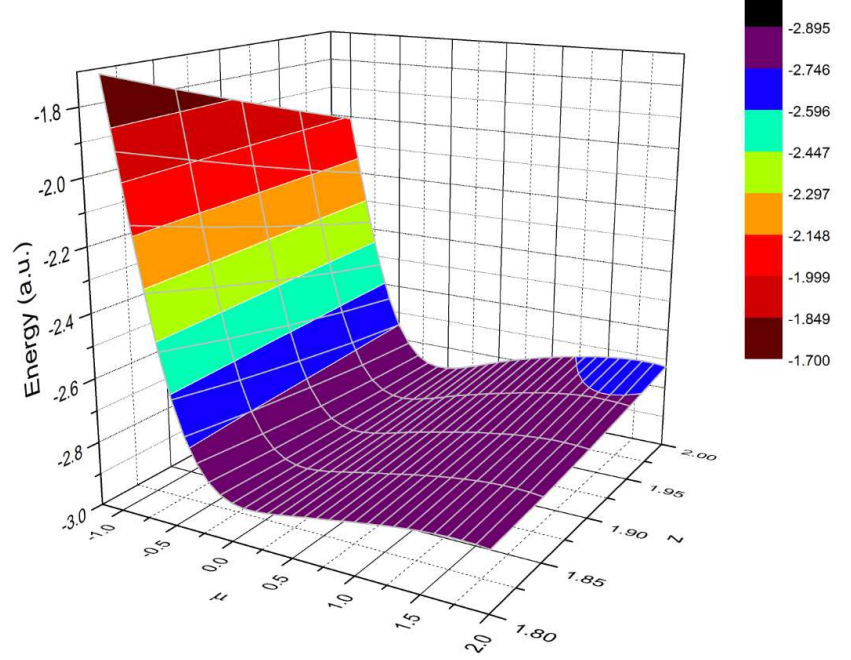

Figure 3. Variation of the ground state energy of helium with respect to the variational parameters, using the third trial wave function. Energy on Zaxis, $\mu$ on $X$-axis and the parameter $Z$ on $Y$-axis.

The variation of the ground state energy of helium with respect to the two variational parameters $Z$ and $\lambda$ of the fourth trial wave function is shown in Figure 4.

\subsection{The Low-Lying Excited States of Helium}

Using the trial wave functions for the excited states of helium, we find that $z_{0}=2$, so that the effective nuclear 
charge is constant for all the excited states. This is may be due to the fact that one of the two electrons is in the $1 s$ state and the other electron is at a higher level. The values of the

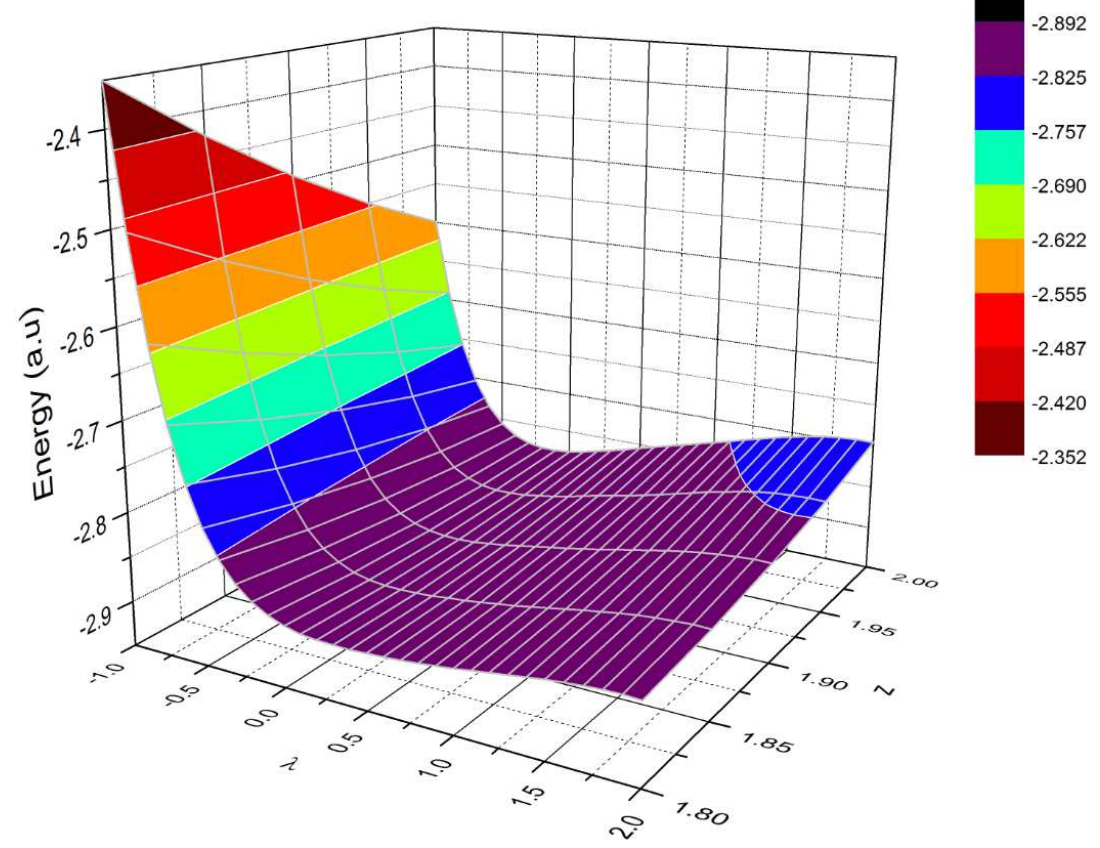

Figure 4. Variation of the ground state energy of the helium atom with respect to the variational parameters, using the fourth trial wave function. Energy on Zaxis, $\lambda$ on $X$-axis and the parameter $z$ on $Y$-axis.

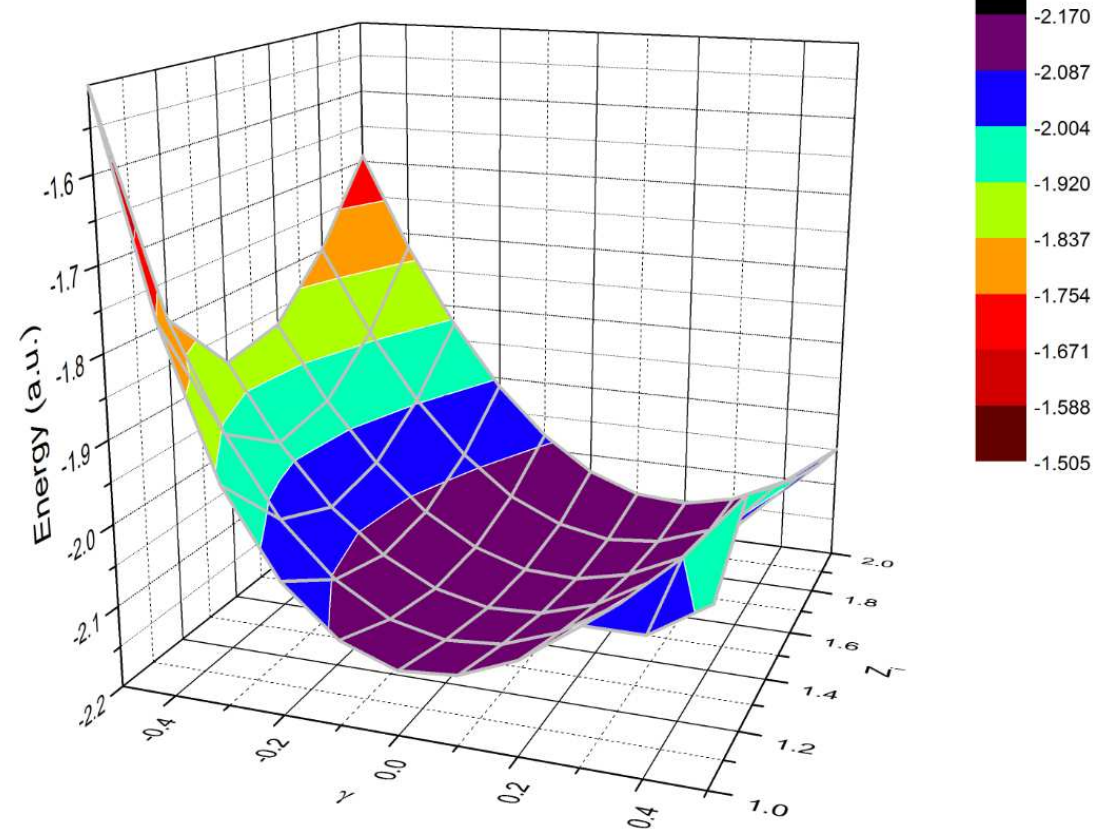

Figure 5. Variation of the energy of the state $2^{3} S$ with respect to the variational parameters, using the first trial wave function. Energy on $Z$-axis, $\lambda$ on $X$-axis and $z$ on $Y$-axis.

Table 3. The best values of the variational parameters for the low-lying excited states of helium using the four trial wave functions.

\begin{tabular}{|c|c|c|c|c|c|}
\hline State & $z_{i}$ & $\gamma$ & $\beta$ & $\mu$ & $\lambda$ \\
\hline $2^{3} \mathrm{~S}$ & 1.620 & 0.10 & 0.30 & 0.100 & 0.300 \\
\hline $2^{1} \mathrm{~S}$ & 0.865 & 0.04 & 0.65 & 0.178 & 0.535 \\
\hline $2^{1} \mathrm{P}$ & 1.000 & 0.03 & 1.00 & 0.200 & 0.690 \\
\hline $2^{3} \mathrm{P}$ & 1.200 & 0.01 & 0.40 & 0.100 & 0.300 \\
\hline
\end{tabular}


The resulting values of the energies of the low-lying excited states of helium are given in Table 4 .

Table 4. Calculated energies $(E)$ with standard deviation $(\sigma)$ compared with exact energies for the low-lying excited states of helium using the different trial wave functions.

\begin{tabular}{llllll}
\hline State & $\boldsymbol{E}^{\mathbf{1}}$ (a. u.) $\boldsymbol{\sigma}$ & $\boldsymbol{E}^{\mathbf{2}}$ (a. u.) $\boldsymbol{\sigma}$ & $\boldsymbol{E}^{\mathbf{3}}$ (a.u.) $\boldsymbol{\sigma}$ & $\boldsymbol{E}^{\mathbf{4}}(\mathbf{a} . \mathbf{u}.) \boldsymbol{\sigma}$ & $\boldsymbol{E}^{\mathbf{5}}$ (a. u.) \\
\hline $2^{3} \mathrm{~S}$ & -2.168946 & -2.168902 & -2.168500 & -2.168533 & -2.1750051 \\
& $1.6 \times 10^{-5}$ & $1.5 \times 10^{-5}$ & $1.6 \times 10^{-5}$ & $1.7 \times 10^{-5}$ & -2.1752293763 \\
$2^{1} \mathrm{~S}$ & -2.145346 & -2.145965 & -2.145213 & -2.145255 & -2.1460 \\
& $2.4 \times 10^{-5}$ & $2.0 \times 10^{-5}$ & $2.1 \times 10^{-5}$ & $2.0 \times 10^{-5}$ & -2.1238431 \\
$2^{1} \mathrm{P}$ & -2.122766 & -2.123401 & -2.12248 & -2.123371 & -2.123842897 \\
& $8.0 \times 10^{-6}$ & $4.0 \times 10^{-6}$ & $5.0 \times 10^{-6}$ & $4.0 \times 10^{-6}$ & -2.1331646 \\
$2^{3} \mathrm{P}$ & -2.130310 & -2.131621 & -2.131104 & -2.131477 & -2.133164074 \\
\hline
\end{tabular}

1. Using $1^{\text {st }}$ correlation. 2. Using $2^{\text {nd }}$ correlation. 3. Using $3^{\text {rd }}$ correlation. 4. Using $4^{\text {th }}$ correlation. 5. Best values [38].

For the dependence of the excited state energies of helium on the used trial wave functions, we present only the case of the first excited state. Accordingly, we present in Figures 5-8 the variations of the energy of the state $2^{3} \mathrm{~S}$ with respect to the parameters, by using the four trial wave functions, respectively. The other three cases are similar.

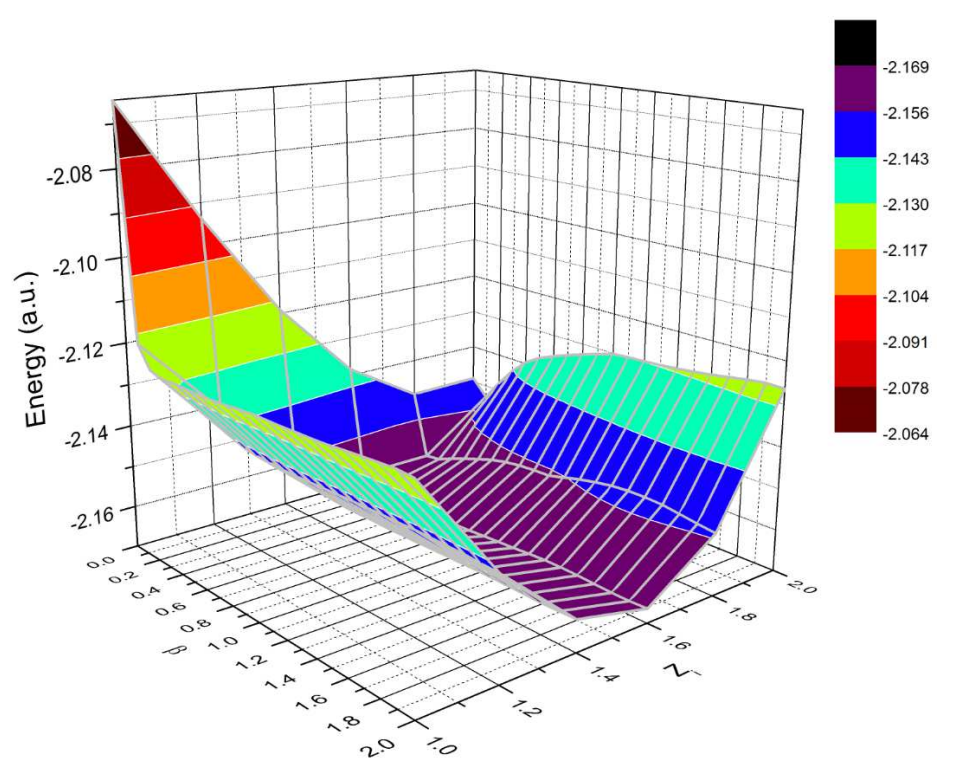

Figure 6. Variation of the energy of the state $2^{3} S$ with respect to the variational parameters, by using the second trial wave function. Energy on $Z$-axis, $\beta$ on $X$ axis and the parameter $z_{i}$ on $Y$-axis.

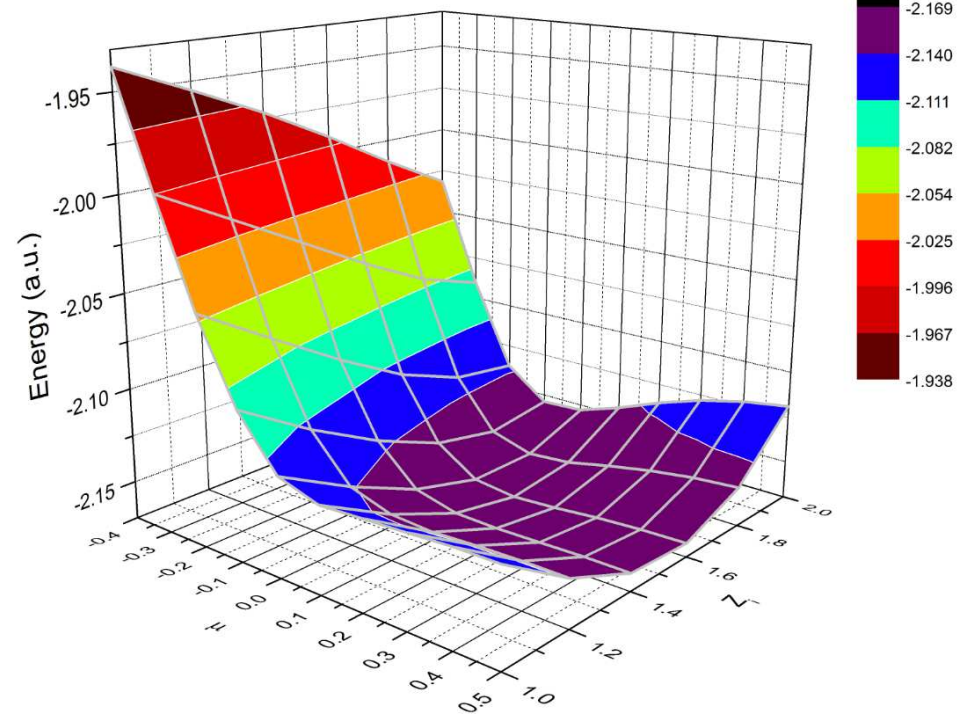

Figure 7. Variation of the energy of the state $2^{3} S$ with respect to the variational parameters, by using the third trial wave function. Energy on $Z$-axis, $\mu$ on $X$ axis and the parameter $z_{i}$ on $Y$-axis. 


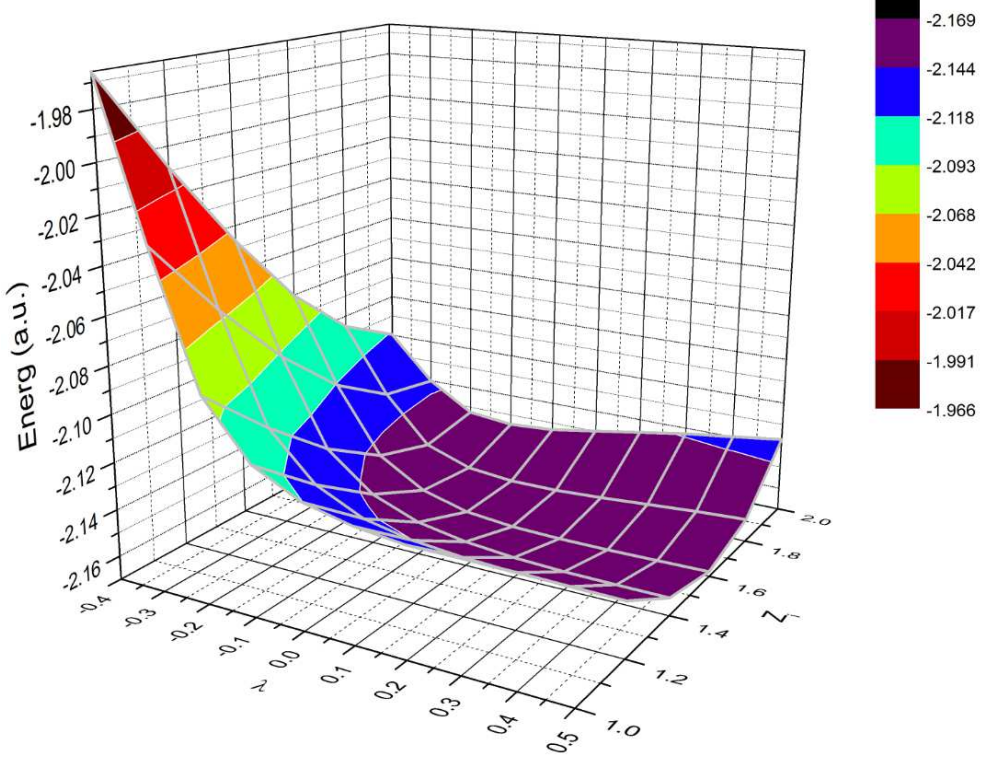

Figure 8. Variation of the energy of the state $2^{3} S$ with respect to the variational parameters, by using the fourth trial wave function. Energy on $Z$-axis, $\lambda$ on $X$ axis and the parameter $z_{i}$ on $Y$-axis.

\subsection{The Ground State of the Confined Helium Atom and Ions}

In this section, we present the results of applying the VMC method to the case of the confined helium atom. The ground state of the confined helium atom, $\mathrm{Li}^{+}$and $\mathrm{Be}^{2+}$ were calculated for different values of the radius $r_{c}$. All energies obtained in atomic units with a set of $10^{8}$ Monte Carlo points to make the statistical error as low as possible.

In Table 5 we present the results obtained for the ground state of the confined helium atom $(Z=2)$ together with the corresponding results available in previous works. The obtained energies calculated for a wide range of values of $r_{c}$. In Table 6 we present the energies of the ground state of the confined $\mathrm{Li}^{+}$as functions of the spherical box radius. Finally, in Table 7 we present the resulting energies of the ground state of the confined $\mathrm{Be}^{2+}$ as functions of the spherical box radius. It seen from these tables that the small values of the spherical box radius $r_{c}$ describe the case of strong confinement where for large values, $r_{c} \geq 3.5$, the compression effect becomes not noticeable and the energy is nearly stable and approaches to the corresponding exact value. Our results are in good agreement, in comparison, with previous data. As the atoms are compressed, they become constrained in a diminishing spherical box so that according to the quantum mechanical uncertainty principle, the electrons increase their momentum and thereby leading to a net gathering of kinetic energy. When the increase in the confinement kinetic energy becomes predominant and cannot be compensated by the increase of the Coulomb attractive energy, the energies of the confined helium atom increase.

Table 5. Energies of the ground state of the confined helium atom as functions of the spherical box radius. All values are in atomic units.

\begin{tabular}{lllll}
\hline $\boldsymbol{r}_{\boldsymbol{c}}$ & Present work & {$[\mathbf{1 5}]$} & {$[\mathbf{3 9 ]}$} & {$[\mathbf{1 7 ]}$} \\
\hline 0.5 & 22.7411 & 22.7413 & 22.7437 & 22.741303 \\
0.6 & 13.3181 & 13.3182 & 13.3204 & 13.318127 \\
\hline
\end{tabular}

\begin{tabular}{lllll}
\hline $\boldsymbol{r}_{\boldsymbol{c}}$ & Present work & {$[\mathbf{1 5}]$} & {$[\mathbf{3 9 ]}$} & {$[\mathbf{1 7 ]}$} \\
\hline 0.8 & 4.6102 & 4.6104 & 4.6125 & 4.610408 \\
1.2 & -0.7087 & -0.7088 & -0.7070 & -0.708802 \\
1.4 & -1.6172 & -1.6173 & -1.6156 & -1.617330 \\
1.8 & -2.4226 & -2.4245 & -2.4230 & - \\
2.5 & -2.8111 & -2.8078 & - & - \\
3.5 & -2.8919 & -2.8936 & - & - \\
4 & -2.8987 & -2.9004 & -2.8988 & -2.900534 \\
5 & -2.9033 & -2.9034 & -2.9020 & -2.903408 \\
6 & -2.9035 & -2.9037 & -2.9024 & -2.903650 \\
\hline
\end{tabular}

Table 6. Energies for the ground state of the confined $\mathrm{Li}^{+}$as functions of the spherical box radius. All values are in atomic units.

\begin{tabular}{llll}
\hline $\boldsymbol{r}_{\boldsymbol{c}}$ & Present work & {$[\mathbf{1 5 ]}$} & {$[\mathbf{3 9 ]}$} \\
\hline 0.5 & 11.76771 & 11.7768 & 11.7790 \\
0.6 & 3.9934290 & 3.9262 & 3.9284 \\
0.8 & -2.893898 & -2.8632 & -2.8612 \\
1.2 & -6.407358 & -6.4065 & -6.4047 \\
1.4 & -6.855290 & -6.8732 & -6.8713 \\
1.8 & -7.192726 & -7.1906 & -7.1880 \\
2.5 & -7.358839 & -7.2740 & - \\
3.5 & -7.280496 & -7.2798 & - \\
4 & -7.27923 & -7.2799 & -7.2783 \\
5 & -7.279204 & -7.2799 & -7.2784 \\
6 & -7.279143 & -7.2799 & -7.2784 \\
\hline
\end{tabular}

Table 7. Energies for the ground state of the confined $B e^{2+}$ as functions of the spherical box radius. All numbers are in atomic units.

\begin{tabular}{llll}
\hline $\boldsymbol{r}_{\boldsymbol{c}}$ & Present work & {$[\mathbf{1 5}]$} & {$[\mathbf{3 9 ]}$} \\
\hline 0.5 & 0.10801980 & 0.1056 & 0.1078 \\
0.6 & -6.288159 & -6.2423 & -6.2402 \\
0.8 & -11.26839 & -11.2679 & -11.2658 \\
1.2 & -13.36396 & -13.3733 & -13.3701 \\
1.4 & -13.556580 & -13.5590 & -13.5552 \\
1.8 & -13.63929 & -13.6449 & -13.6415 \\
2.5 & -13.65413 & -13.6553 & - \\
\hline
\end{tabular}




\begin{tabular}{llll}
\hline $\boldsymbol{r}_{\boldsymbol{c}}$ & Present work & {$[\mathbf{1 5}]$} & {$[\mathbf{3 9 ]}$} \\
\hline 3.5 & -13.65678 & -13.6555 & - \\
4 & -13.6514 & -13.6555 & -13.6539 \\
5 & -13.657630 & -13.6555 & -13.6519 \\
6 & -13.65711 & -13.6555 & -13.6539 \\
\hline
\end{tabular}

It can be concluded that our results for helium ions $(Z=3$, 4) for all values of $r_{c}$ exhibit good accuracy comparing with the other data. When the nuclear charge increase, the Coulomb attraction between the nucleus and the electron becomes stronger, which keeps the electrons moving ever closer to the nucleus and then lead to a more compact atom. The rate of energy increases speeds up as the nuclear charge of the atom increase.

\subsection{The Ground State of Helium Atom in a Strong Magnetic Field}

The VMC method is employed to calculate the groundstate energies of the helium atom in a magnetic field regime between $0-100$ a.u. Our consideration focused on the ground state of the helium atom by using the four trial wave functions. All energies are obtained in atomic units, with a set of $10^{8}$ Monte Carlo integration points to make the statistical error as low as possible. Table 8 shows the calculated energies for some magnetic field strength between $0-100$ a. $u$ using the four trial wave functions.

Table 8. Calculated energies $(E)$ with standard deviation $(\sigma)$ for the ground state of helium in a strong magnetic field by using the four trial wave functions. Previous results [26]. and [40] are also shown.

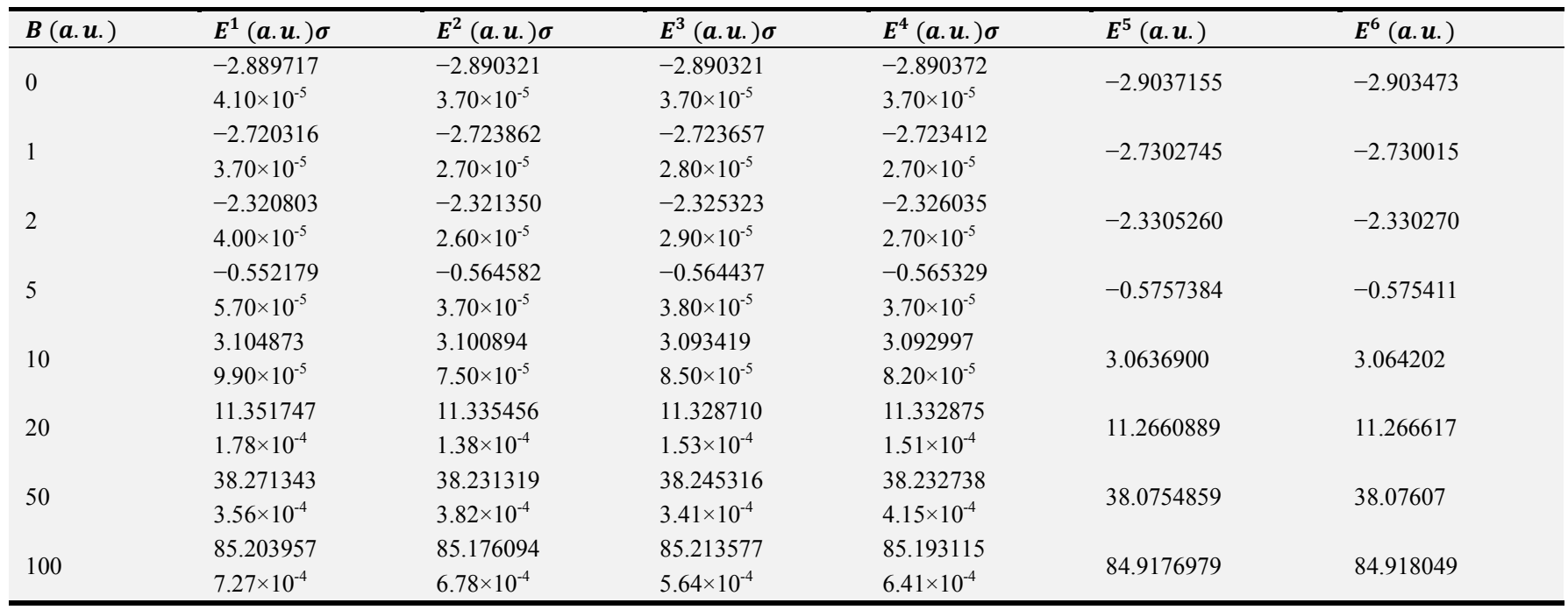

1. Using $1^{\text {st }}$ correlation. 2. Using $2^{\text {nd }}$ correlation. 3. Using $3^{\text {rd }}$ correlation. 4. Using $4^{\text {th }}$ correlation. 5. Previous results [26]. 6. Previous results [40].

\section{Conclusions}

The trial wave function for the ground state of the helium atom and helium like ions, which considers the cusp conditions, gives results in excellent agreement with the corresponding exact values.

The obtained excited state energies of helium, by using the second trial wave function, are also in good agreement with the previous results.

For the excited states of helium, when the two electrons are located in different shells, the electron in the $1 s$ orbit is affected by the nucleus charge so that the parameter $z_{0}$ is equal to $Z$ (nuclear charge), while the $z_{i}$ parameter of the other electron is considered to be a variational parameter.

The VMC method was employed to study the helium atom when it is compressed by a spherical box, for various values of the spherical box radii. In the case of small values of $r_{c}$, which describes the strong compression, as well as the case of large values of $r_{c}$, the calculated energies for both helium and its isoelectronic ions $\mathrm{Li}^{+}$and $\mathrm{Be}^{2+}$ are also in good agreement with the previous results.

The obtained energies of the helium atom in a strong magnetic field, by using our trial wave functions, are in good agreement with the best values, calculated in previous studies for helium in a strong magnetic field.

\section{References}

[1] J. E. Lynn, I. Tews, S. Gandolfi, and A. Lovato, "Quantum Monte Carlo Methods in Nuclear Physics: Recent Advances," Annu. Rev. Nucl. Part. Sci., 2019.

[2] N. Metropolis, A. W. Rosenbluth, M. N. Rosenbluth, A. H. Teller, and E. Teller, "Equation of state calculations by fast computing machines," J. Chem. Phys., vol. 21, no. 6, pp. 1087-1092, 1953.

[3] U. Kleinekathöfer, S. H. Patil, K. T. Tang, and J. P. Toennies, "Boundary-condition-determined wave function for the ground state of helium and isoelectronic ions," Phys. Rev. A At. Mol. Opt. Phys., 1996.

[4] H. Nakashima and H. Nakatsuji, "Solving the electron-nuclear Schrödinger equation of helium atom and its isoelectronic ions with the free iterative-complement-interaction method," J. Chem. Phys., 2008. 
[5] C. Le Sech, "Accurate analytic wavefunctions for twoelectron atoms," J. Phys. B At. Mol. Opt. Phys., 1997.

[6] D. Bressanini and G. Morosi, "A compact boundarycondition- determined wavefunction for two-electron atomic systems," J. Phys. B At. Mol. Opt. Phys., 2008.

[7] R. S. Chauhan and M. K. Harbola, "Improved le Sech wavefunctions for two-electron atomic systems," Chem. Phys. Lett., 2015.

[8] A. J. Thakkar and T. Koga, "Variational calculations for helium-like ions using generalized Kinoshita-type expansions," Theor. Chem. Acc., 2003.

[9] R. Habrovský, "An explicitly correlated helium wave function in hyperspherical coordinates," Chem. Phys. Lett., 2018.

[10] E. G. Drukarev, M. Y. Amusia, E. Z. Liverts, R. Krivec, and V. B. Mandelzweig, "Wavefunctions of helium-like systems in limiting regions," J. Exp. Theor. Phys., 2006.

[11] A. W. King, A. L. Baskerville, and H. Cox, "Hartree-Fock implementation using a Laguerre-based wave function for the ground state and correlation energies of two-electron atoms," Philos. Trans. R. Soc. A Math. Phys. Eng. Sci., 2018.

[12] H. E. Montgomery, J. Katriel, and K. D. Sen, "Asymptotic behavior of two-electron expectation values in two-electron excited states," Phys. Lett. Sect. A Gen. At. Solid State Phys., vol. 383 , no. 36 , p. $126007,2019$.

[13] J. Li, N. D. Drummond, P. Schuck, and V. Olevano, "Comparing many-body approaches against the helium atom exact solution,” SciPost Phys., vol. 6, no. 4, pp. 1-37, 2019.

[14] M. Mostafanejad, "Structure of free complement wavefunction for the ground and the first excited state of helium atom," J. Theor. Comput. Chem., vol. 16, no. 6, pp. 1-14, 2017.

[15] A. Flores-Riveros and A. Rodríguez-Contreras, "Compression effects in helium-like atoms $(Z=1, \ldots, 5)$ constrained by hard spherical walls," Phys. Lett. Sect. A Gen. At. Solid State Phys., vol. 372, no. 40, pp. 6175-6182, 2008.

[16] X. Wen-Fang, "A Study of Confined Helium Atom," Commun. Theor. Phys., 2007.

[17] C. Laughlin and S. I. Chu, "A highly accurate study of a helium atom under pressure," J. Phys. A Math. Theor., 2009.

[18] H. E. Montgomery, N. Aquino, and A. Flores-Riveros, "The ground state energy of a helium atom under strong confinement," Phys. Lett. Sect. A Gen. At. Solid State Phys., 2010.

[19] M. Rodriguez-Bautista, C. Díaz-García, A. M. NavarreteLópez, R. Vargas, and J. Garza, "Roothaan's approach to solve the Hartree-Fock equations for atoms confined by soft walls: Basis set with correct asymptotic behavior," J. Chem. Phys., vol. 143, no. 3, 2015.

[20] M. Rodriguez-Bautista, R. Vargas, N. Aquino, and J. Garza, "Electron-density delocalization in many-electron atoms confined by penetrable walls: A Hartree-Fock study," Int. J. Quantum Chem., vol. 118, no. 13, pp. 1-11, 2018.

[21] T. D. Young, R. Vargas, and J. Garza, “A Hartree-Fock study of the confined helium atom: Local and global basis set approaches," Phys. Lett. Sect. A Gen. At. Solid State Phys., vol. 380, no. 5-6, pp. 712-717, 2016.

[22] E. Ley Koo, "Recent Progress in Confined Atoms and
Molecules: Superintegrability and Symmetry Breakings," Rev. Mex. Física, vol. 64, no. 4, p. 326, 2018.

[23] J. Katriel, H. E. Montgomery, A. Sarsa, and E. Buendía, "Hund's rule in open-shell states of two-electron systems: From free through confined and screened atoms, to quantum dots," Nanosyst. Physics, Chem. Math., vol. 10, no. 1, pp. 31-41, 2019.

[24] Á. Luzón, E. Buendía, and F. J. Gálvez, “One and two body densities for excited states of the helium confined atom," Int. J. Quantum Chem., no. August, pp. 1-12, 2019.

[25] R. Vargas, "Shannon Entropy for the Hydrogen Atom Confined by Four Different Potentials,” pp. 208-218, 2019.

[26] X. Wang, J. Zhao, and H. Qiao, "Helium atom in strong magnetic fields: An application of the configurationinteraction method with Hylleraas-Gaussian basis," Phys. Rev. A - At. Mol. Opt. Phys., 2009.

[27] S. Boblest, C. Schimeczek, and G. Wunner, "Ground states of helium to neon and their ions in strong magnetic fields," Phys. Rev. A - At. Mol. Opt. Phys., 2014.

[28] W. Zhu and S. B. Trickey, “Accurate and balanced anisotropic Gaussian type orbital basis sets for atoms in strong magnetic fields,” J. Chem. Phys., vol. 147, no. 24, 2017.

[29] A. Zafar, E. Martin, and S. Shannon, "High resolution magnetic field measurements in hydrogen and helium plasmas using active laser spectroscopy," Rev. Sci. Instrum., vol. 89, no. 10 , pp. $1-5,2018$.

[30] S. B. Doma, M. O. Shaker, A. M. Farag, and F. N. ElGammal, "Ground states of helium atom and hydrogen negative ion in the presence of magnetic field using variational Monte Carlo technique," Acta Phys. Pol. A, 2014.

[31] S. B. Doma and F. N. El-Gammal, "Application of variational Monte Carlo method to the confined helium atom," J. Theor. Appl. Phys., 2012.

[32] S. Doma, M. Shaker, A. Farag, and F. El-Gammal, "Excited states of helium atom in a strong magnetic field using variational Monte Carlo technique," Indian J. Phys., 2018.

[33] S. B. Doma, M. O. Shaker, A. M. Farag, and F. N. ElGammal, "Variational Monte Carlo calculations of lithium atom in strong magnetic field," J. Exp. Theor. Phys., 2017.

[34] S. B. Doma and F. N. El-Gammal, "Atomic properties of the two-electron system using variational Monte Carlo technique," Acta Phys. Pol. A, 2012.

[35] T. M. Whitehead, M. H. Michael, and G. J. Conduit, "Jastrow correlation factor for periodic systems," Phys. Rev. B, 2016.

[36] J. O. Hirschfelder, "Removal of electron-electron poles from many-electron hamiltonians," J. Chem. Phys., 1963.

[37] A. Banerjee, C. Kamal, and A. Chowdhury, "Calculation of ground- and excited-state energies of confined helium atom," Phys. Lett. Sect. A Gen. At. Solid State Phys., 2006.

[38] J. L. Marin and S. A. Cruz, "Enclosed quantum systems: Use of the direct variational method," J. Phys. B At. Mol. Opt. Phys., 1991.

[39] E. V. Ludeña and M. Gregori, "Configuration interaction calculations for two-electron atoms in a spherical box," J. Chem. Phys., 1979. 
[40] X. Wang and H. Qiao, "Configuration-interaction method with Hylleraas-Gaussian-type basis functions in cylindrical coordinates: Helium atom in a strong magnetic field," Phys. Rev. A - At. Mol. Opt. Phys., 2008. 